\title{
Reconstruction Techniques for Image Analysis of Ancient Islamic Mosaics
}

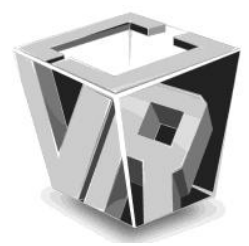

\author{
Francisco Albert Gil ${ }^{1}$, José María Gomis ${ }^{1}$ and Manuel Pérez ${ }^{2}$ \\ ${ }^{I}$ Department of Graphics Engineering, Universitat Politècnica de València, Spain \\ ${ }^{2}$ Department of Engineering Design, Universidade de Vigo, Spain
}

\begin{abstract}
This paper presents a methodology for tile image reconstruction. This methodology is integrated in a computer tool that allows automatic image analysis. Both, the reconstruction and analysis techniques are based on vectorial tools, the application of the theory of symmetry groups and perceptual psychology. Image analysis allows us to obtain information about design patterns which is used to restitute (recover missing motifs), unify (choose between different motifs that should be equal) and standardize (fit motifs exactly to their symmetry axes and rotation centers) old designs. This methodology for tile image reconstruction has been applied to different Islamic mosaics from the Alhambra of Granada (Spain), the Aq-Saray Palace of Shar-i-Sabz (Uzbekistan) and the Red Fortress of Agra (India). The analysis results are presented in this paper.
\end{abstract}

Index Terms-Islamic mosaics, 2D Reconstruction, Theory of symmetry groups, Image processing.

\section{INTRODUCTION}

Traditional tile pattern designs consist of motifs which are combined using geometrical transformations to fill the space of the tiling. The different ways of combining these transformations and, therefore, all possible symmetries of the design pattern, are limited and have been described in the theory of symmetry groups [1][2]. The different motifs together with their plane symmetry group (design structure) provide all the information of the design pattern.

Ancient Islamic mosaics constitute a historical heritage consisting of tiles that were manufactured using old tiling techniques. Pattern design recovery and image processing are of great importance for different purposes, like exhibitions of our cultural heritage, media art, cultural restoration or, even, design pattern reuse in the tile industry. With this in mind, the following tasks must be carried out:

1) The morphological and syntactic analysis of the designs, inherent in the images of tiles.

2) Reconstruction and recovery of the design's theoretical conception, overcoming the consequences of the deterioration of the tiles caused both by time (breakages, cracks, etc.) and by the techniques used in the past (shapes which should be the same, but are not, etc.).

The images under analysis present the following common problems:

Manuscript received on March 5, 2008

E-mails: fraalgi1@dig.upv.es
1) They come from historical sources, often with centuries of antiquity, and of course, they can be deteriorated (see detail in Fig. 1).

2) The tiles were hand-made, thus the distribution of the tile design elements (objects and motifs) does not accurately match the design pattern. That is to say, the geometric transformations (translations, rotations and symmetries) indicated by the plane symmetry group are not exactly equal for all the elements of the image.

3) The hand-craft character of the tiles causes discrepancies between object shapes that, in their theoretical conception, should be equal (see Fig. 2).

4) In addition to this, during image analysis, when image segmentation is complex, mainly if there is noise and low contrast, the original image errors are emphasized (see Fig. 3).

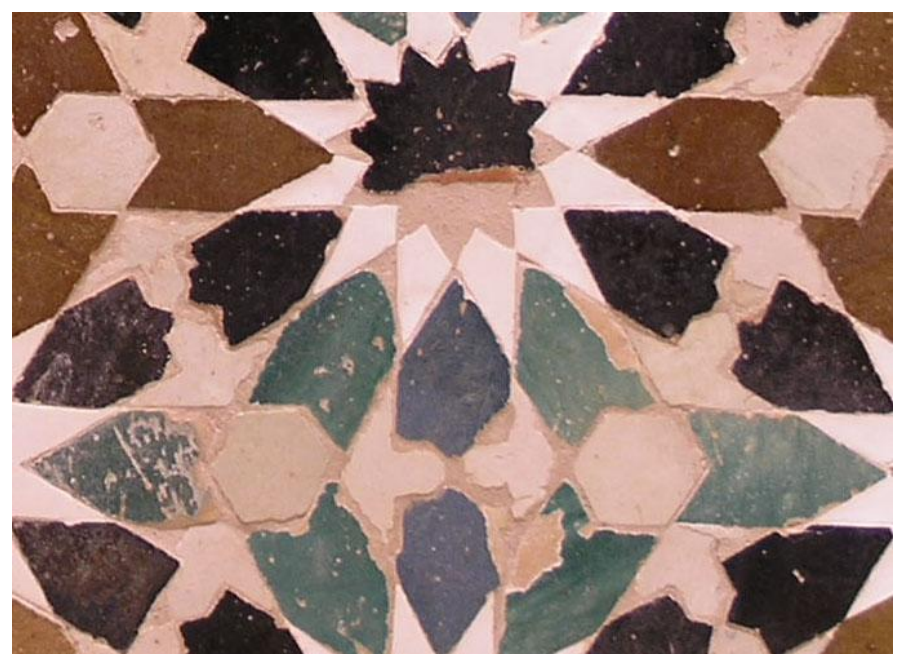

Fig. 1. Damaged Islamic mosaic $\left(14^{\text {th }}\right.$ century). Source: the Alhambra's museum
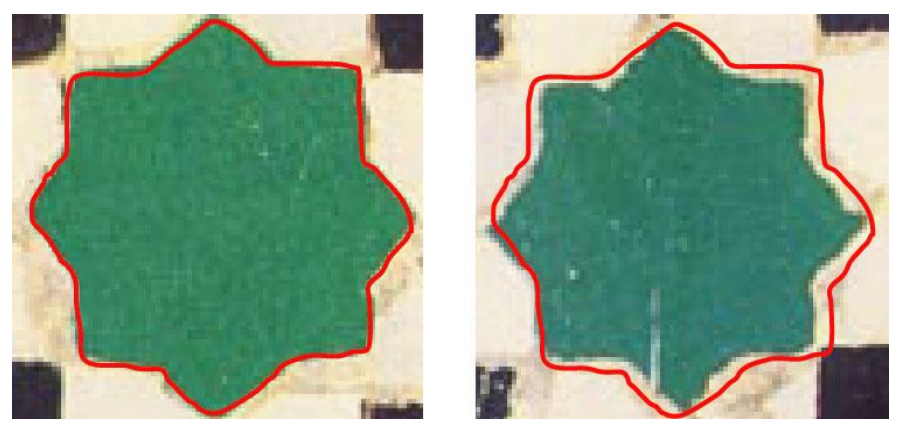

Fig. 2. Discrepancies between equal object shapes 

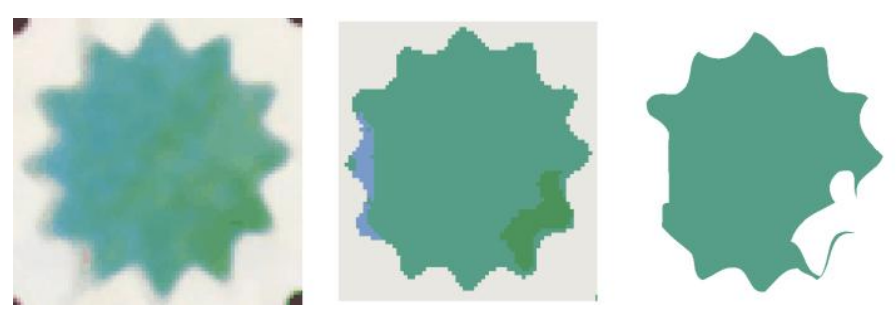

Fig. 3. 8-end star design segmented into three different objects due to hue differences

\section{RECONSTRUCTION TECHNIQUES}

Image reconstruction is a common topic in image processing, although the applications of those techniques differ from the particular application presented in this paper. For example, specific reconstruction techniques are used to recover 3D data from projections [3], shades [4] or stereoscopic vision [5]. Other techniques attempt to recover an image that has been degraded by a process modeled as a linear position-invariant function followed by additive noise [6] or images distorted by nonlinear transformations [7], although these techniques always work at a the level of pixels rather than at the level of objects or object groupings; furthermore, tile degradation (due to cracking, stains, ...) does not occur uniformly (the reconstruction models are generally developed to solve errors from electronic devices like sensors or digitizers). This lack of uniformity in the degradation process is balanced with design pattern uniformity; and the theory of symmetry groups can provide the necessary information for design pattern reconstruction.

The design reconstruction techniques proposed in this paper use the data obtained from an image analysis process developed by the authors. These techniques can be divided into three types:

1) Restitution: recovering missing elements.

2) Unification: choosing among different elements related to each other by a geometric transformation.

3) Standardization: generating the object shape or its arrangement within a motif, as well as the distribution of motifs in the design pattern to accurately match their symmetry axes and rotation centers.

In this paper, we describe the way these reconstruction techniques are carried out throughout the image analysis process, using the tile image of figure 1 as an illustration. In the following section, the general methodology of image analysis is described at a functional level (input/output). And, in the following sections the reconstruction techniques (by motifs, by the fundamental parallelogram and by the plane symmetry group) are described in detail.

\section{IMAGE ANALYSIS}

Artificial vision tools can be used to analyze the tile images. However for further steps in the image analysis process other more sophisticated tools are required [8][9][10]. These tools are based on the theory of symmetry groups [1][2], which constitutes the grammar or, more properly, the mathematical basis of the model.

In Fig. 4 (ordered from top to bottom and left to right) the different operational spaces of the analysis tool, corresponding to different information levels, can be observed. The first is a

bip map space and the rest are vectorial spaces. The analysis corresponds to the Islamic mosaic shown in Fig. 1.

\subsection{Image Space}

The pixels are grouped to form objects. This is common to all computer vision applications; two basic stages [11][12] are image preprocessing (noise elimination and contrast enhancement) and segmentation (grouping of pixels according to their environmental features).

The output information is an object list with the following attributes:

1) Region: set of points that form the object.

2) Color: average color of all the points of the region.
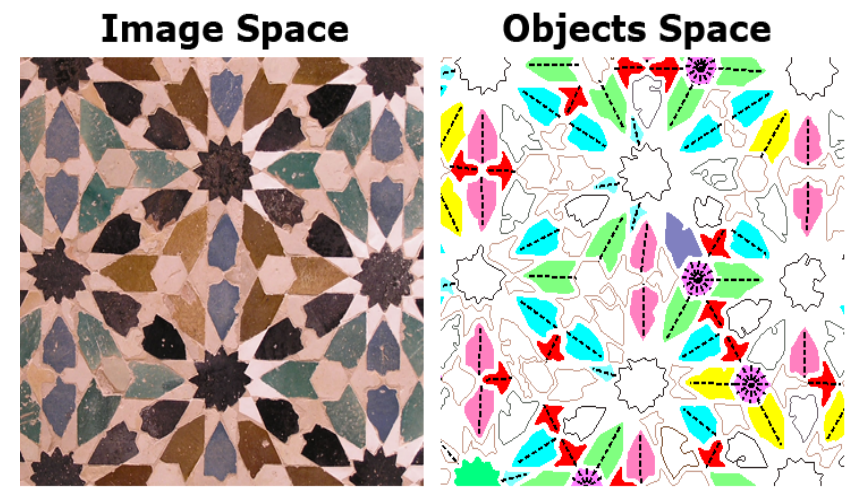

Motifs Space

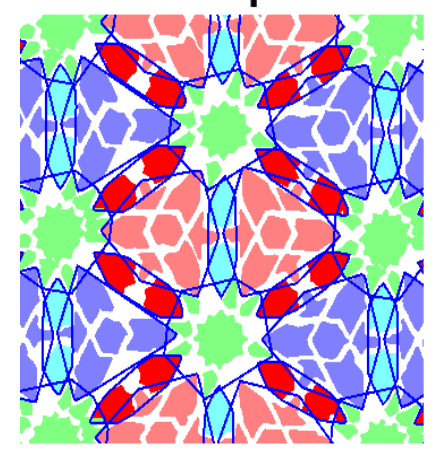

Fig. 4. Steps of the Analysis Tool

\subsection{Objects Space}

The contours delimiting the region are vectorized (expressed as compound Bezier curves). With this representation the copy and geometric transformation of the objects for image reconstruction is easier. By means of scan-line algorithms the representation of a region, organized by lines, can be recovered, and in this way the verifications of superposition made in the reconstruction processes become more efficient.

Taking the external contour as a representation of the object's shape and its centroid as the origin of the reference system, object comparison is performed [13][14] in order to classify the objects, taking into account their color. The object's comparison process calculates the object isometries (rotation or reflection) that relate equal objects. Likewise, different objects are compared, comparing each one with itself and analyzing the coincidences. Their symmetries (both rotation and reflection) are thereby obtained. 
The output data is another list of objects, each one with the following attributes:

1) Shape: color, area, centroid and set of vectorized contours.

2) Class: class number and displacement, rotation or reflection with respect to an object of the class considered as reference object.

3) Symmetry: point symmetry group and, with specular symmetry, angle of an axis.

In the objects space of Fig. 4, the objects of each class are represented with the same color; the symmetry axes have also been drawn.

\subsection{Motifs Space}

Here the objects are grouped following perceptual criteria such as cocircularity, inclusion and proximity [15][16]. These motifs are compared considering the objects that they contain and how they are distributed within the motifs (existence of a predominant isometry between equal objects) and their symmetries are calculated analyzing the isometries existing between the objects of the motif. Like for the objects, the isometry that relates equal motifs is calculated and it will be the predominant isometry of their objects.

The output data is a list of motifs, added to the list of objects, each one with the following attributes:

1. Composition: objects contained in the motif.

2. Shape: minimum convex polygon that contains all the objects of the motif.

3. Class: class number and rotation or reflection with respect to a motif of the class considered as representative.

4. Symmetry: point symmetry group and, with rotational symmetry, angle of an axis.

In Fig. 4, in the motifs space, the objects within motifs of the same class have the same color. Each motif is represented by a contour line surrounding its objects.

\subsection{Structure Space}

The relationship between equal motifs is analyzed to determine the isometries fulfilled in the whole design pattern [8][9]. The translations that form the fundamental parallelogram are obtained from the motifs with the same orientation. The centers of rotation and symmetry axes determining the plane symmetry group have been obtained from the motifs rotated or reflected, respectively.

The output information, added to object and motif lists, is:

1) Fundamental parallelogram: two vectors representing its sides.

2) Plane symmetry group: identifier and indication of which side or diagonal is parallel to the symmetry axes (in groups PG, PM and PMG).

\section{RECONSTRUCTION BY MEANS OF MOTIFS}

In Fig. 5 the objects of each motif have been enclosed within a contour. Some motifs are incomplete, for example:

1) Many objects are missing in motif A located on the upper left corner.

2) Motif B is of the same class as motif A, although it has less missing objects.

3) In motif $\mathrm{C}$ two objects arrowhead-shaped are missing.
After motif comparison we count with enough data to correct image defects. This image correction, or reconstruction, can be of two types:

1) Restitution: to insert the missing objects in a motif bringing them from another motif of the same class.

2) Unification: to make equal different objects located in the same position as their respective motif.

The steps to follow are:

1) To identify which objects (and their respective class) occupy the same position within motifs of the same class; then apply to the objects the transformation that relates the motifs and calculate the overlapping regions. This is the moment to select the most suitable kind of pattern design reconstruction: restitution (no overlapping), unification (overlapping with objects of other classes) or none (overlapping with objects of the same class).

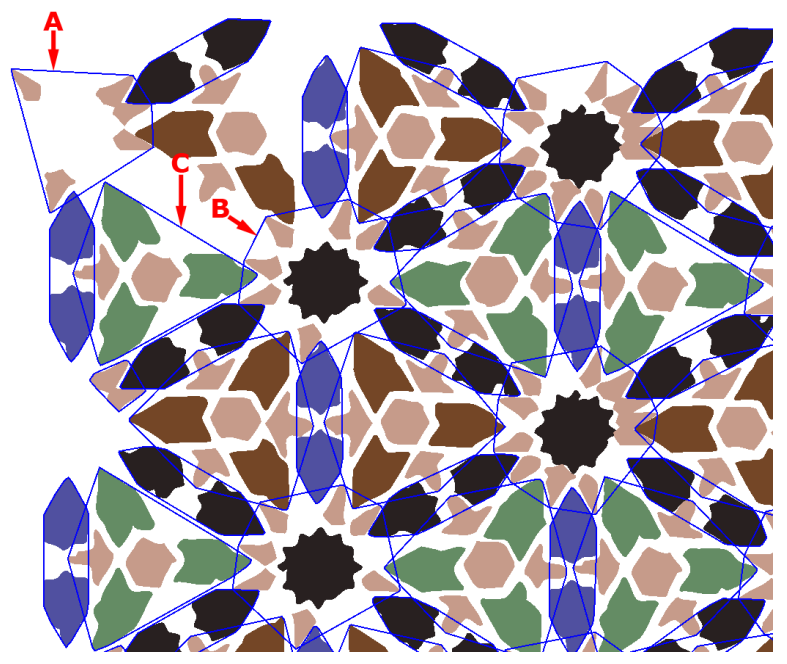

Fig. 5. Design before motif reconstruction

2) The remaining objects must be chosen in the cases where unification is necessary. Two simple criteria are: the object belonging to a class with more members or the object whose region covers a larger area. The non-chosen objects will be eliminated.

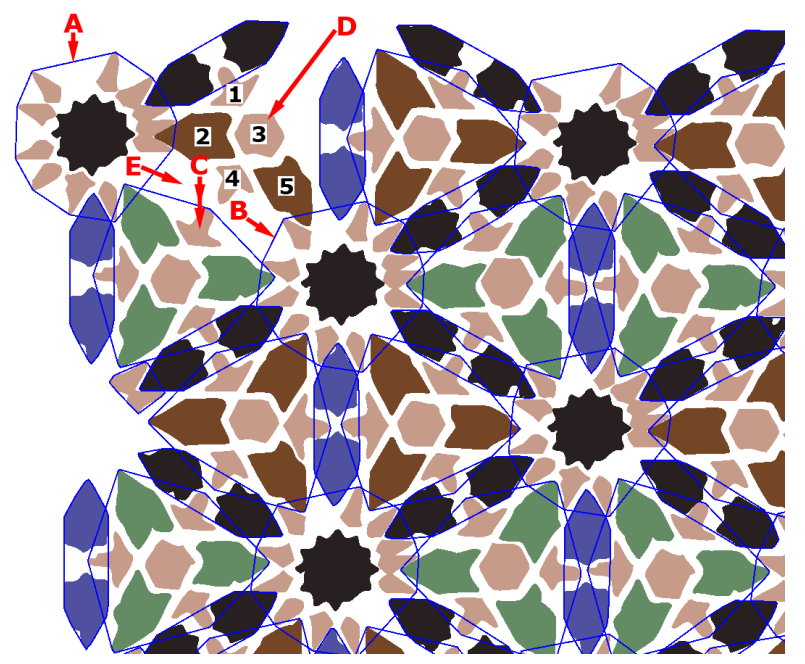

Fig. 6. Design after motif reconstruction 
3) Finally, in the case of both restitution and unification, objects will be copied from some motifs to others by means of duplication followed by the application of the existing isometry between motifs.

In Fig. 6 we can see the result of motif reconstruction: the motifs of each class are all equal. Motifs A, B and C have new objects from other motifs of the same class. The star-shaped motifs (A and B) still have missing objects, which will be recovered at a later stage.

\section{V.RECONSTRUCTION BY MEANS OF THE FUNDAMENTAL PARALLELOGRAM}

Once the fundamental parallelogram has been obtained, we have new information to reconstruct the design pattern. The repetition of fundamental parallelograms will form a mesh and all the motifs located in the same relative position within each cell of the mesh must be equal. The corresponding isometry relates the motifs in a linear combination of the two translations defined by the sides of the parallelogram.

Objects without a motif are considered as motifs with one single object, and the regions used in the calculation of overlapping will be those of all the objects of the motif.

Fig. 6 shows some defects that can be corrected in the process of pattern design reconstruction using the fundamental parallelogram:

1) There are five objects $(1,2,3,4,5)$ instead of a motif (they have not been grouped).

2) In the area marked by the leader line, E should have a motif.

In Fig. 7 we can observe the fundamental parallelogram and the reconstruction process carried out:

1) Unification: the non-grouped objects have been replaced by the corresponding motif (D).

2) Restitution: the missing motif $E$, has been restored.

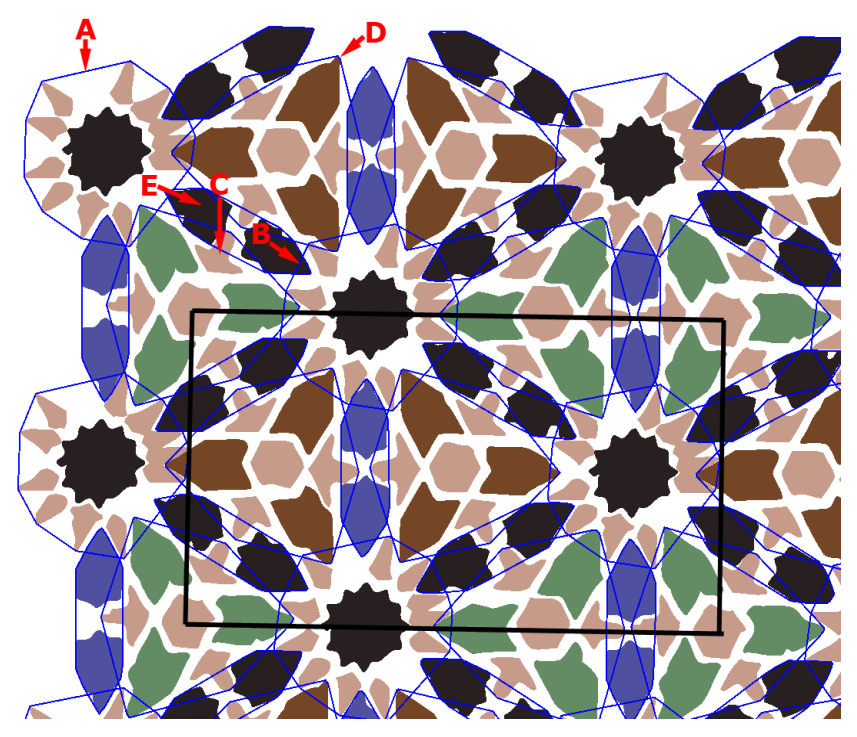

Fig. 7. Design after reconstruction by using the fundamental parallelogram

\section{RECONSTRUCTION BY MEANS OF THE PLANE SYMMETRY GROUP}

Once the plane symmetry group is obtained, the isometries will be "distributed" in the following way:

1) For each motif we will distinguish between the internal isometries (IIM: non-slide symmetry axes which pass through their centroid and centers of rotation coinciding with their centroid) and external (EIM: the remainder). EIMs will indicate which isometries can be applied to the motif to match equal motifs.

2) For each object we will use the IIMs of its motif dividing them into internal and external to the object (IIO and EIO). EIOs indicate which isometries have to be applied to the object to match equal objects within the same motif

The information concerning IIM and EIM allows us to carry out the reconstruction of the motifs and design pattern at content level. In Fig. 8 we can observe the symmetry axes and centers of rotation of the design (simplified) and the result of the design reconstruction process.

In order to restitute and unify the contents of each motif, we will use its objects and its IIM. In Fig. 8, the star-shaped motifs have been reconstructed through object restitution.

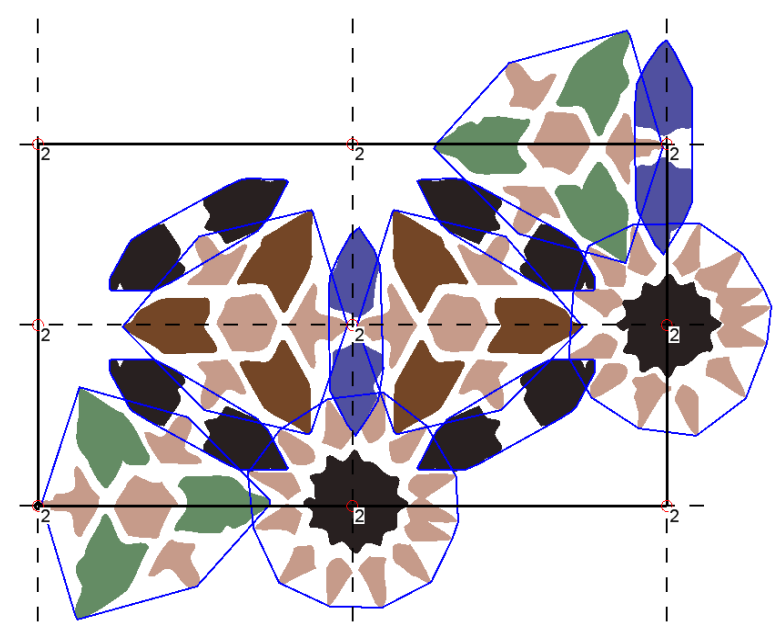

Fig. 8. Design after pattern reconstruction (contents) by means of the plane symmetry group

In order to reconstruct the content of the design, we use the motifs and their EIM. In the example, this has not been necessary.

The information concerning the internal symmetry axes and centers of rotation of the objects can be useful to standardize object representation. The process followed is: first, the regions of the objects of the same class are superimposed and averaged. Following this, the regions are regenerated taking account of the transformations of its point symmetry group. Finally, a vectorization of the contour portion between consecutive symmetry axes (reflection symmetry), or within a sector (rotational symmetry), is carried out. This process is replicated according to the corresponding isometries.

In Fig. 9 the 8 different shape types are shown both before (upper) and after (lower) standardization.

The motifs can be standardized using their IIM, in the same way as the objects have been standardized. The difference being 
that motif standardization does not consist of the redistribution of the region's points, but rather of the objects of the motif. For each set of objects corresponding to each other by the internal motif isometries we will:

1) Compute the average distance from object centroids to motif centroid.

2) Choose a representative object and eliminate the rest.

3) Apply the representative to the average distance calculated.

4) If a symmetry axis passes through it, rotate the object to align it with the axis.

5) Generate the rest of the objects by copy and application of isometries.

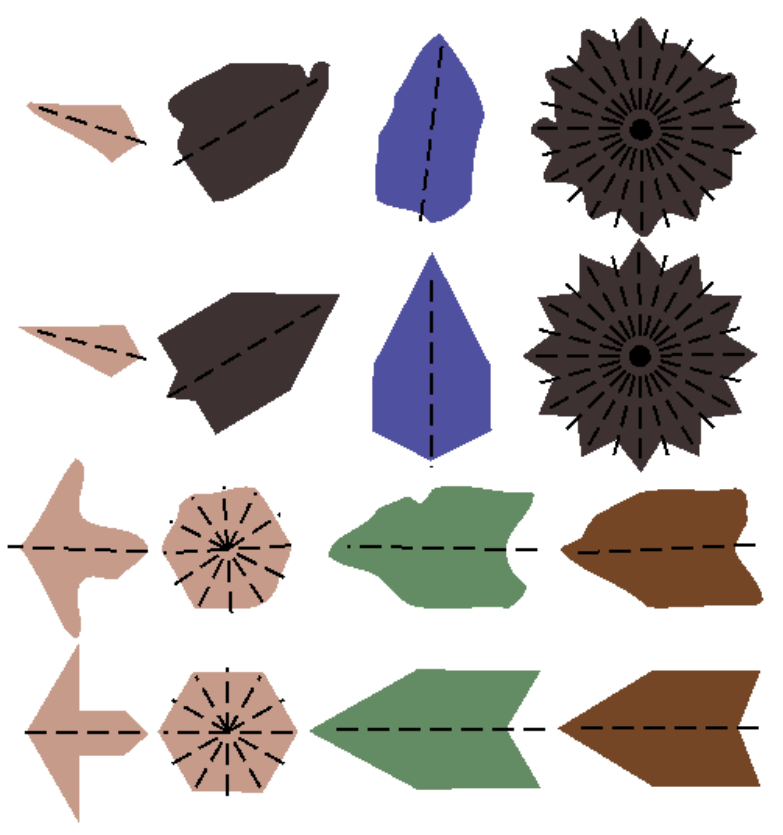

Fig. 9. Objects before and after standardization

In Fig. 10 we can observe the symmetry axes of each motif, as well as the motifs before (upper image) and after (lower image) motif standardization. It fits its objects perfectly well.

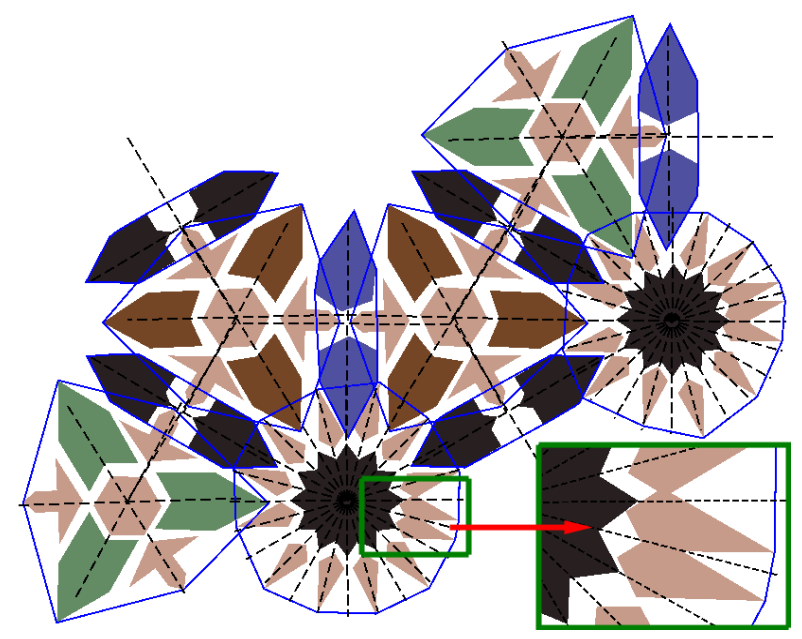

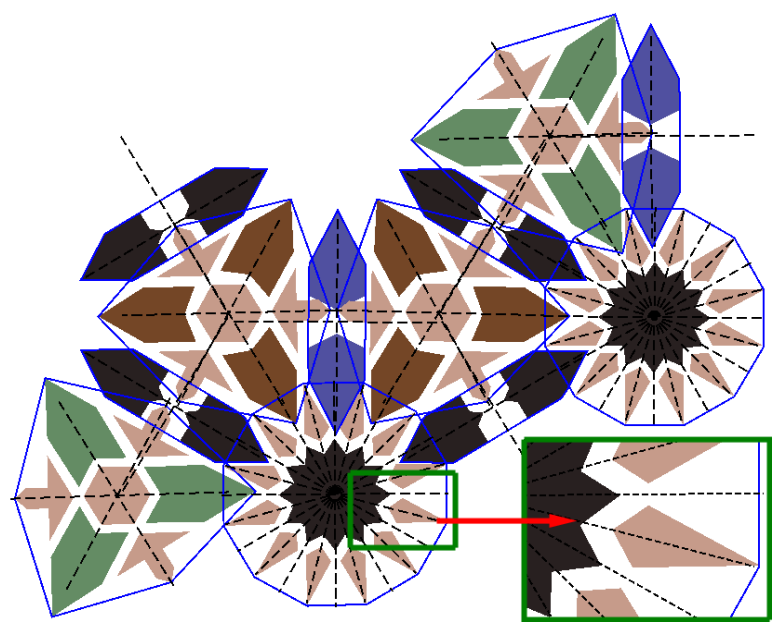

Fig. 10. Motifs before and after motif standardization

Finally, we also apply this methodology to standardize the structure, distributing the motifs according to their EIM:

1) The motifs on a rotation center are centered in this center by means of a translation.

2) The motifs which have an internal axis are rotated to fit to it.

In Fig. 11 the design before (upper image) and after (lower image) structure standardization can be observed. The symmetry axes of the motif match perfectly well those of the design.

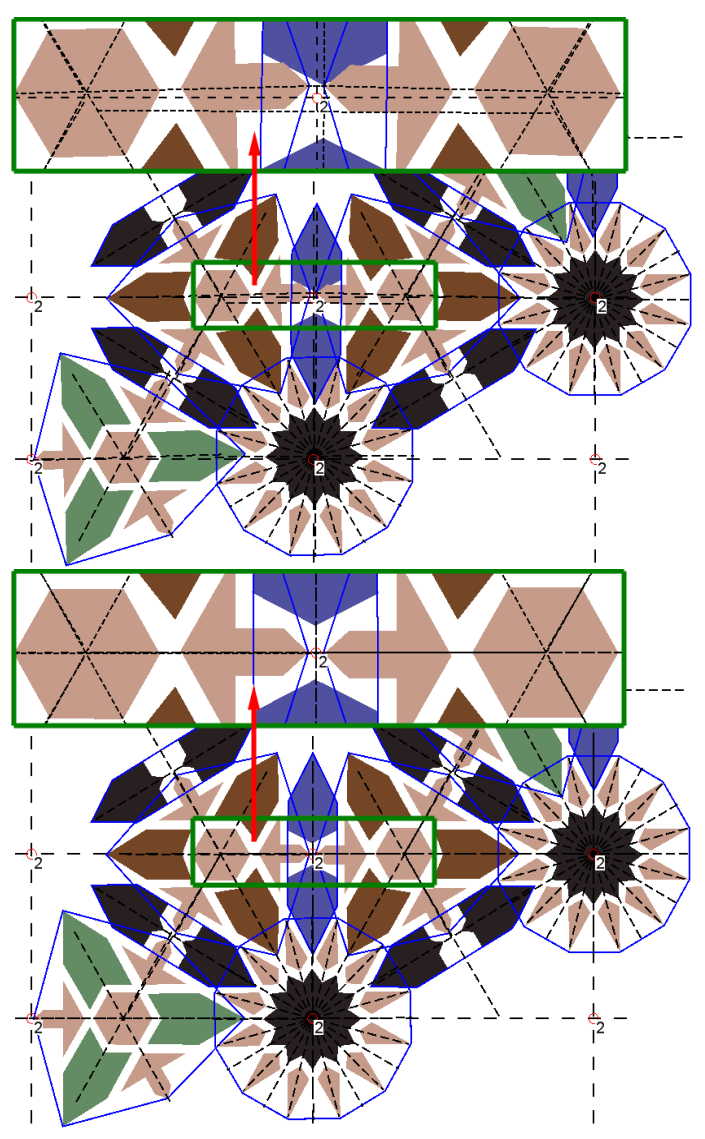

Fig. 11. Design before and after structure standardization 


\section{RESULTS}

The described reconstruction techniques have been implemented in a computer tool developed by the authors to analyze tile images. These tools have been used successfully to analyze and reconstruct different tile mosaics from three of the most important Islamic architectures in the world: the Alhambra of Granada (Spain), the Timur Lang's Aq-Saray Palace of Shar-i-Sabz (Uzbekistan) and the Akbar's Red Fortress of Agra (India).

In Fig. 12 we can see two details of the original image (above) and of the image of the analyzed and reconstructed design (below) used as an example.

In figures 13, 14, 16 and 17 details of the original images and of the analyzed and reconstructed designs of Islamic mosaics from the Aq-Saray Palace and the Red Fortress can be observed.

Finally, in order to show other possibilities of the analysis and reconstruction tools presented in this work, in figure 15 we have included the analysis and reconstruction of a Jali (filigree stone carving) Geometry from an ancient Mogul Palace in India.
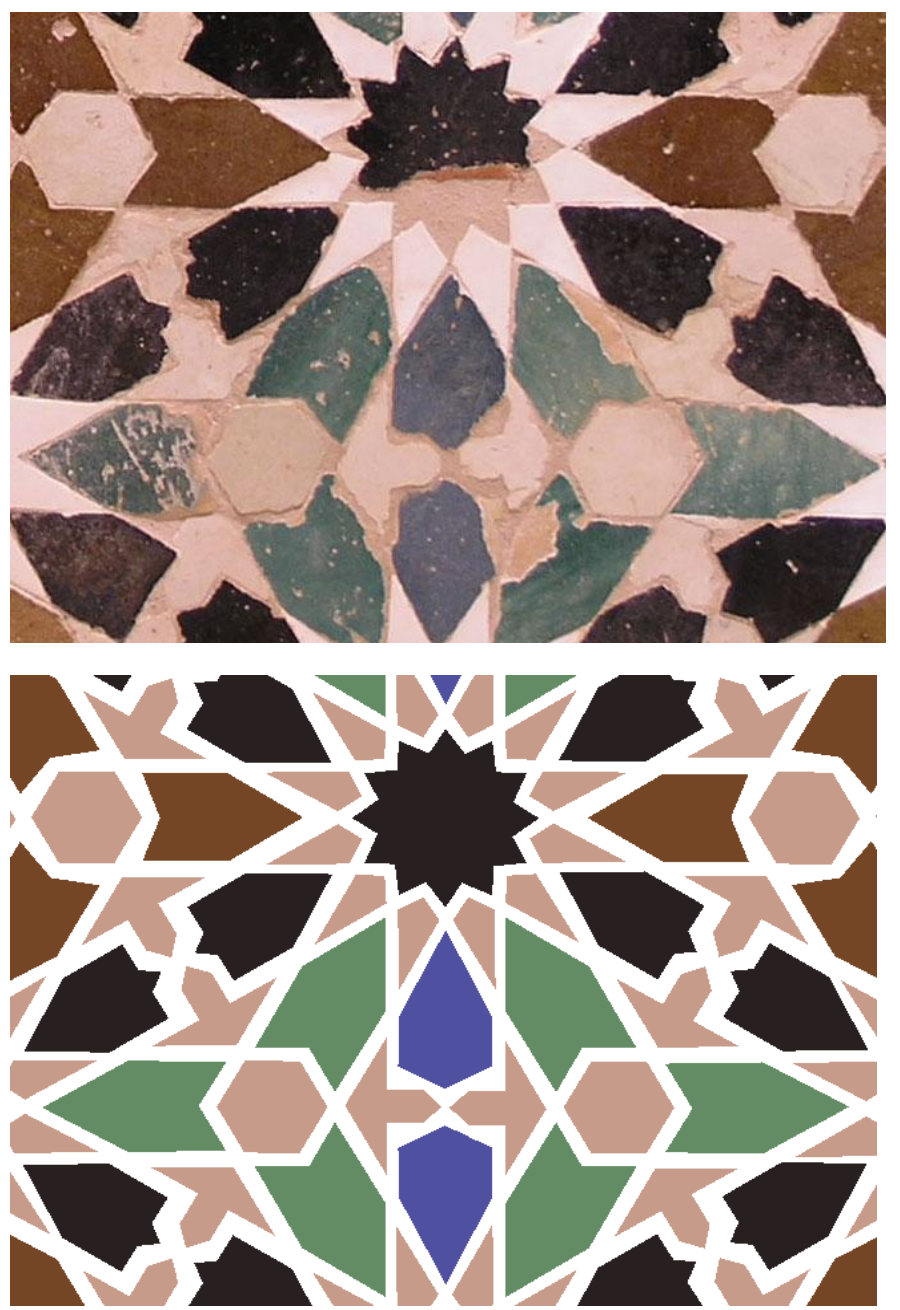

Fig. 12. Details of the original image and final design after analysis and reconstruction
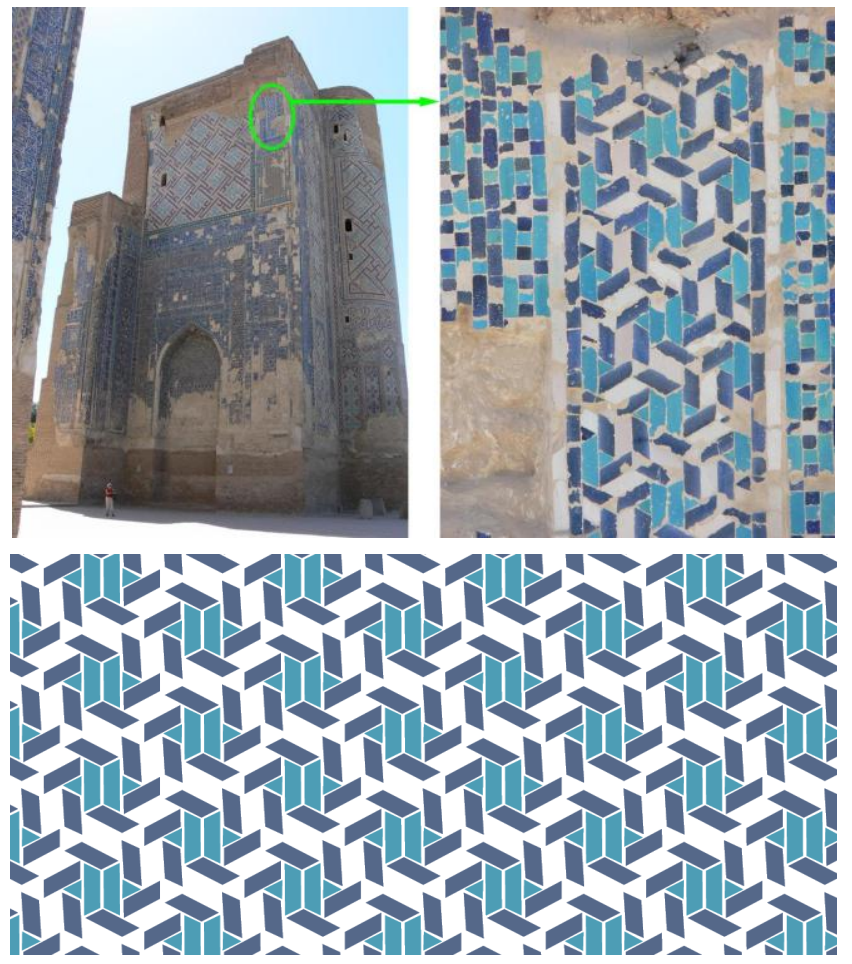

Fig.13. Location (on the jamb of a destroyed arch), detail and reconstruction of an Islamic mosaic. Aq-Saray Palace of Shar-i-Sabz ( $14^{\text {th }}$ century).
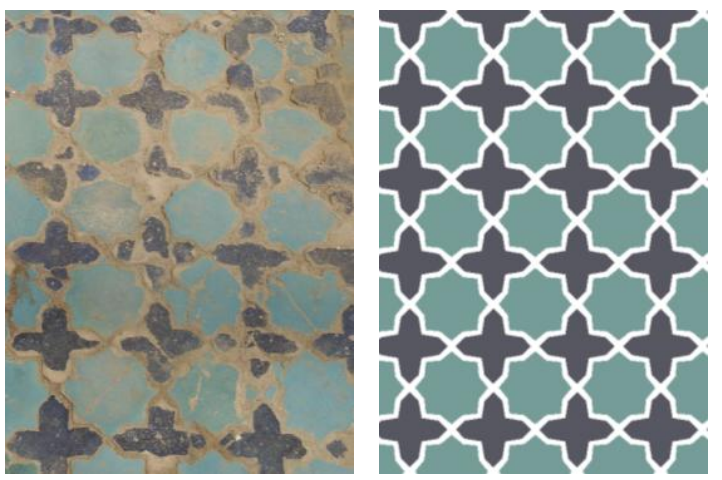

Fig. 14. Ancient pavement of Aq-Saray Palace (left) and Reconstruction (right)
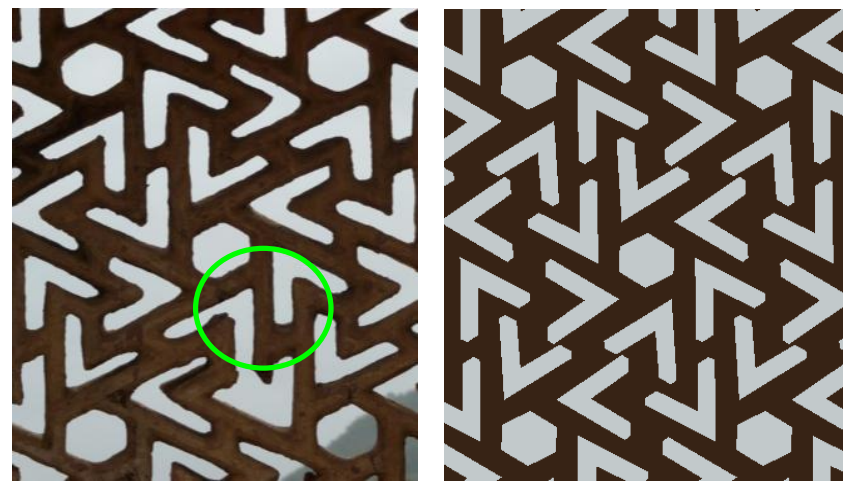

Fig. 15. Original image of a broken Jali (left) and Geometry reconstruction (right) 

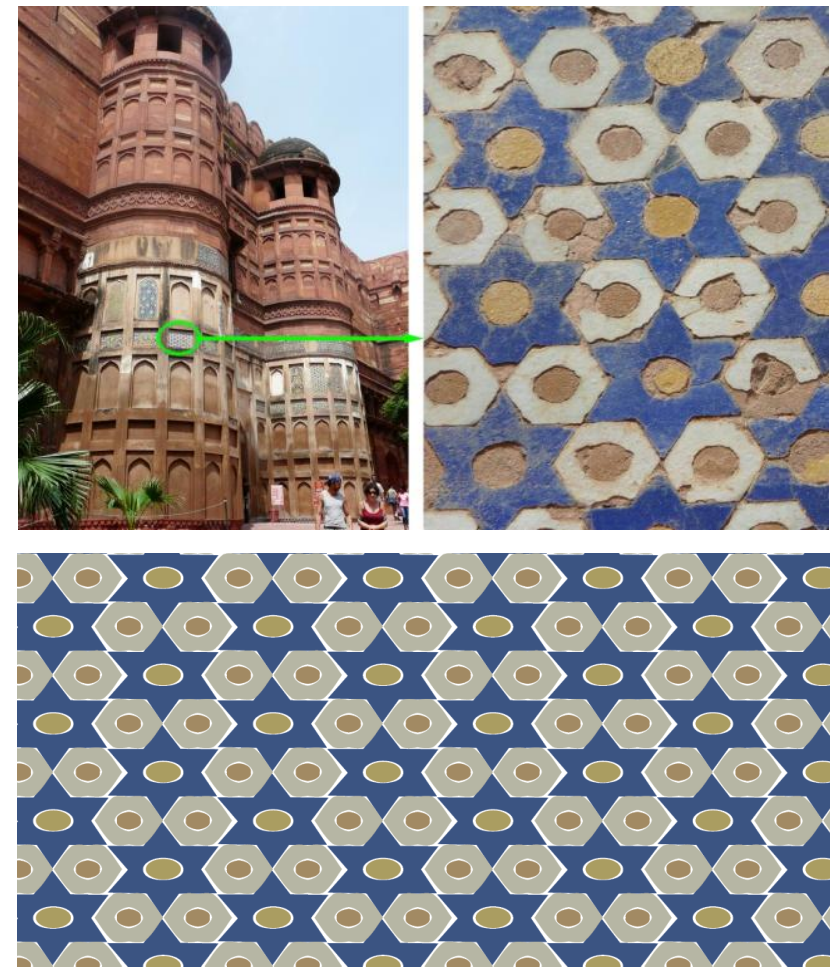

Fig. 16. Location, detail and reconstruction of an Islamic mosaic. Red fortress of Agra ( $16^{\text {th }}$ century)
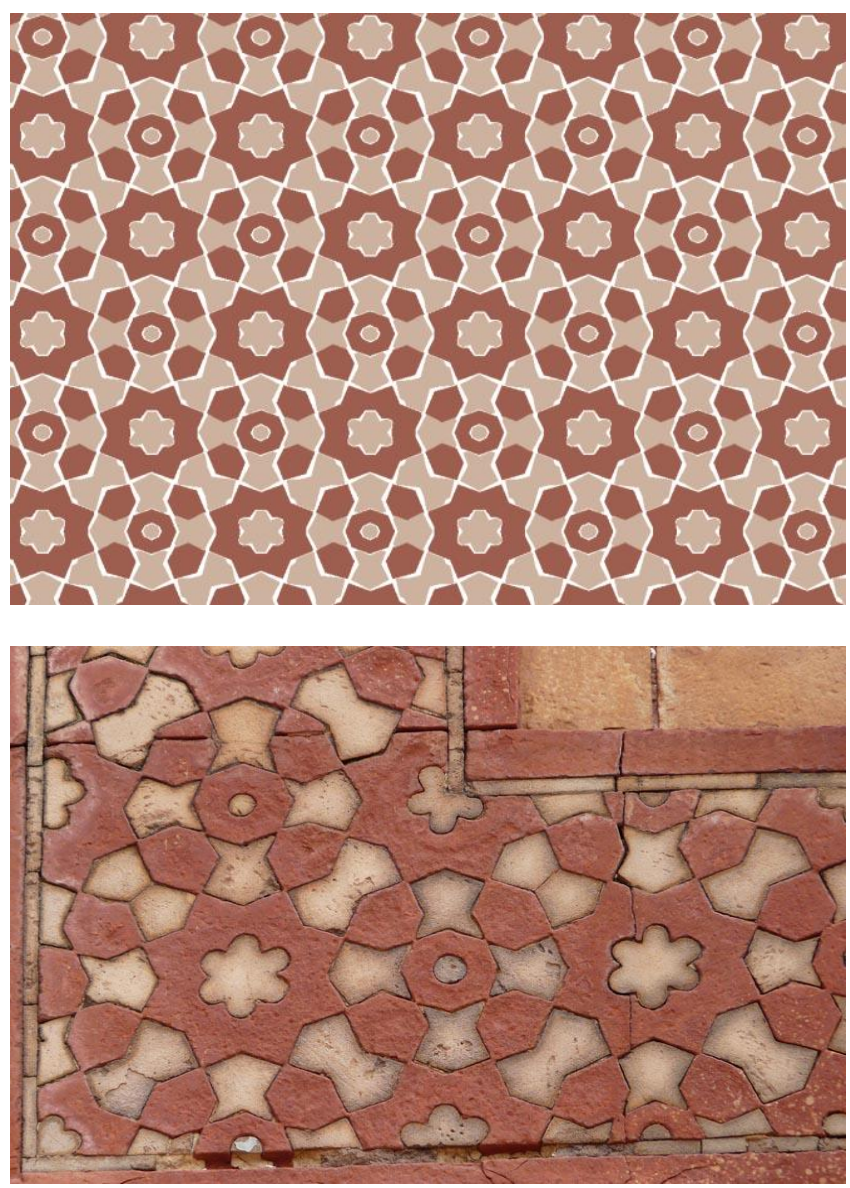

Fig. 17. Original image and reconstruction of an Islamic mosaic. Red fortress of Agra

\section{CONCLUSIONS}

In this article a set of techniques for the reconstruction of tile images has been described. This allows the recovery of damaged parts together with improvement of the exactitude of tile's geometrical pattern.

The techniques used are progressively applied according to the stage in the design analysis process, and relative information of motifs, fundamental parallelogram and plane symmetry group which organize the image will be extracted. This information has been used like a context in the same way as the parts of a text can be interpreted through the context.

The final analysis results allow the arrangement of all information relative to the theoretical conception of the tile's design.

\section{REFERENCES}

[1] B. Grünbaum, and G.C. Shephard, Tilings and Patterns, W. H. Freeman, New York, 1987.

[2] D. Schattschneider, "The Plane Symmetry Groups: Their Recognition and Notation", The American Mathematical Monthly, 85, 1978, pp. 439-450.

[3] P. Parodi, G. Piccioli, "3D Shape Reconstruction by Using Vanishing Points". IEEE transactions on pattern analysis and machine intelligence, vol. 18 , no. 2, 1996, p. 211-217

[4] A. Stewart, M. Langer, "Toward Accurate Recovery of Shape from Shading Under Diffuse Lighting". IEEE transactions on pattern analysis and machine intelligence, vol. 19, no. 9, 1997, p. 1020-1025

[5] A. Dipanda, S. Woo, F. Marzani, et al. "3-D Shape Reconstruction in an Active Stereo Vision System Using Genetic Algorithms". Pattern recognition, vol. 36, no. 9, 2003, p. 2143-2157

[6] K. Castleman, Digital Image Processing, 2nd ed. Prentice Hall, Upper Saddle River, N. J, 1996.

[7] E. S. Kim, S. Lee, Y. Tang, "Nonlinear shape restoration of distorted images with Coons transformation". Pattern recognition, vol. 29, no. 2, 1996, p. 217-230

[8] M. Valor, F. Albert, J.M. Martí, and M. Contero, "Textile and Tile Pattern Design Cataloguing Using Automatic Detection of the Plane Symmetry Group", Proceedings of the 2003 Computer Graphics Int. (CGI), IEEE Computer Society Press, Tokyo, 2003, pp. 112-119.

[9] M. Valor, F. Albert, J.M. Gomis, and M. Contero, "Analysis Tool for Cataloguing Textile and Tile Pattern Designs", Proceedings of the II Int. Workshop on Computer Graphics and Geometric Modeling (CGGM), Lecture Notes in Computer Science, Montreal, 2003, pp. 569-578.

[10] J.M. Valiente, F. Albert, and J.M. Gomis, "Feature Extraction and Classification of Textile Images: Towards a Design Information System for the Textile Industry", Proceedings of the II International Workshop on Pattern Recognition in Information Systems (PRIS), ICEIS Press, Alicante (Spain), 2002, pp. 77-94.

[11] R.C. González, and P. Wintz, Digital Image Processing, Addison-Wesley, 1987.

[12] J.M. Valiente, M. Carretero, J.M. Gomis, and F. Albert, "Image Processing Tool for the Purpose of Textile Fabric Modeling", Proceedings of the XII ADM International Conference on Design Tools and Methods in Industrial Engineering, Rimini (Italy), 2001, pp. 147.

[13] P.J. Van Otterloo, A contour-oriented approach to shape analysis, Prentice-Hall International, 1991.

[14] F. Albert, J.M. Gomis, J.M. Valiente, and M. Carretero, "Object Comparison in Structural Analysis of Decorative Pattern in Textile Design", Proceedings of the XII ADM International Conference on Design Tools and Methods in Industrial Engineering, Rimini (Italy), 2001, pp. 77.

[15] M. Wertheimer, "Laws of Organization in Perceptual Forms", A Source Book of Gestalt Psychology, W. Ellis, 1950, pp. 71-88.

[16] S. Palmer, Vision Science. Photons to Phenomenology, MIT Press, Cambridge (Massachusetts), 2002. 


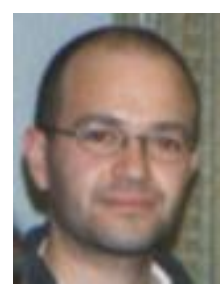

Francisco Albert is a Professor of CAD, 3D animation and Graphic Design with the Graphic Engineering Department at the Universitat Politècnica de València. He earned a degree in Computer Science in 1998, a MS degree in CAD/CAM/CIM in 2000, and his PhD in 2006, all of them from UPV. His research interests focus on graphic pattern analysis, reconstruction and design, and development of spatial abilities using new technologies.

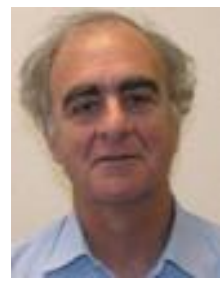

Jose Maria Gomis is a Full Professor of CAD and Graphic Design with the Graphic Engineering Department at the Universitat Politècnica de València (UPV). Industrial Engineering, hi earned his PhD in 1987 from UPV. His research interests focus on graphic pattern analysis, 2D reconstruction and design, and 3D reconstruction in Graphic Engineering.

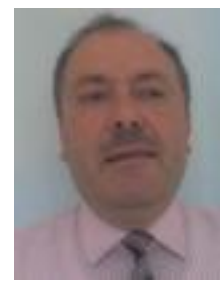

Manuel Pérez is a 1986 Industrial Engineering graduate of the Universidad Politécnica de Madrid, Spain. He received Ph.D. degree from Universidade de Vigo in 1997. Since 1990 he's teaching Graphics at Deparment of Design. His research interests include graphic lenguage, conceptual models for Graphic Engineering, virtual reality applications in product comunication, visualization, design, 3-D models, user interface, image analysis, rendering and modelling, interactive graphics

and animation. 